\title{
Towards topological spectroscopy in the electron microscope with atomic resolution
}

Juan Carlos Idrobo ${ }^{1}$, Andrew R. Lupini ${ }^{2}$, Tracy C. Lovejoy ${ }^{3}$, Nickolay V. Lavrik ${ }^{1}$, Jordan A. Hachtel ${ }^{1}$, Ján Rusz ${ }^{4}$, Niklas Dellby ${ }^{3}$, and Ondrej L. Krivanek ${ }^{3}$

1. Center for Nanophase Materials Sciences, Oak Ridge National Laboratory, Oak Ridge, TN, USA.

2. Materials Science and Technology Division, Oak Ridge National Laboratory, Oak Ridge, TN, USA.

3. Nion Company, 11511 NE 118th St, Kirkland, WA, USA.

4. Department of Physics and Astronomy, Uppsala University, Box 516, S-751 20 Uppsala, Sweden

It has been almost a decade since the first experimental proof that electron beams carrying orbital angular momentum (OAM) can be produced in an electron microscope [1]. Since then, there has been considerable interest, reflected both in the number of publications and the funding of research programs, to utilize sub-nanometer and atomic-size electron beams with OAM for practical imaging and spectroscopy measurements. Yet, so far most of the research in this area has been restricted to describing the possible applications of what could be achieved with electron beams with OAM (also known as vortex beams) rather than performing the actual experiments.

The main limiting factors to bringing vortex beams into the mainstream of the microscopy community is the difficulty of selecting and isolating individual vortex beams. Different approaches have been pursued, such as utilizing holographic masks with dislocations, [2,3] aberration correctors [4,5], and magnetic needles [6]. Images with atomic resolution utilizing vortex beams have been produced [7,8], but the quality of the images indicates that the coherence of the electron beams is not yet optimal, particularly when trying to perform atomically resolved electron magnetic chiral dichroism (EMCD) spectroscopy measurements.

In this talk, I will discuss how and why vortex beams can be utilized to perform novel spectroscopy associated with broken symmetries, such as magnetism. Additionally, I will demonstrate how to produce atomically resolved EMCD measurements utilizing aberrated probes formed by a linear combination of vortex beams (shown schematically in Figure 1) [9]). Finally, I will highlight the current efforts in selecting isolated, atomic-size and coherent vortex beams in ORNL's Nion HERMES [10].

[1] M Uchida and A Tonomura, Nature 464, (2010), p.737.

[2] J Verbeeck, H. Tian, and P. Schattschneider, Nature 467, (2010), p. 301.

[3] BJ McMorran, et al., Science 331, (2011), p.192.

[4] L Clark, et al., Phys. Rev. Lett. 111, (2013), p. 064801.

[5] JC Idrobo, et al., Adv. Strut. Chem Imag. 2, (2016), p. 5.

[6] A Béché, et al., Nature Physics 10, (2014), p. 26.

[7] D Pohl, et al. Scientific Reports 7, (2017) p. 934.

[8] A Béché, et al., Ultramicroscopy 178, (2017), p. 12.

[9] J Rusz and JC Idrobo, Phys. Rev. B, 94, (2016), p. 144430.

[10] Research supported by ORNL's Center for Nanophase Materials Sciences, which is a U.S. Department of Energy Office of Science User Facility (J.C.I. and J.A.H.), and the Materials Sciences and Engineering Division, Office of Basic Energy Sciences, U.S. Department of Energy (A.R.L.), by ORNL's Laboratory Directed Research and Development Program, managed by UT-Battelle, LLC, for the U.S. Department of Energy (J.C.I. A.R.L., J.A.H., N.V.L.), and instrumentation within ORNL's 
Materials Characterization Core provided by UT-Battelle, LLC under Contract No. DE-AC0500OR22725 with the U.S. Department of Energy. J.R. acknowledges funding from Swedish Research Council, Göran Gustafsson's foundation and Carl Tryggers foundation.
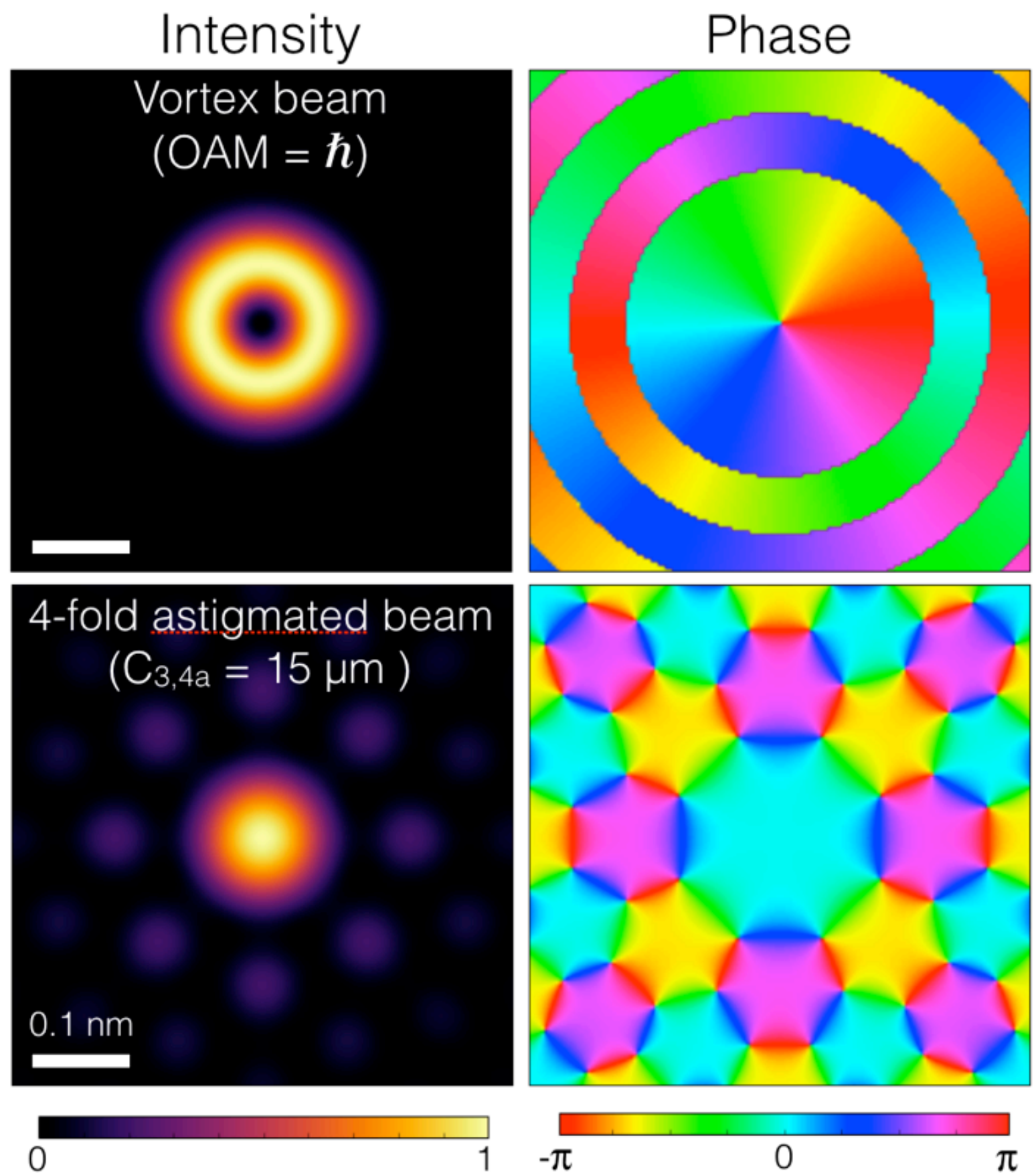

Figure 1. Calculations of an electron vortex beam carrying OAM (top panels) and a fourfold astigmatic electron beam (bottom panels). The calculations were performed using an acceleration voltage of 100 $\mathrm{kV}$ and a convergence semiangle $\alpha=30 \mathrm{mrad}$. Notice that the phase singularity in the vortex beams is also present at the nodes of the fourfold aberrated probe (Figure adapted from Ref. 9). 